PROCEEDINGS OF THE

AMERICAN MATHEMATICAL SOCIETY

Volume 130, Number 4, Pages 935-937

S 0002-9939(01)06155-X

Article electronically published on September 14, 2001

\title{
A QUESTION OF B. PLOTKIN ABOUT THE SEMIGROUP OF ENDOMORPHISMS OF A FREE GROUP
}

\author{
EDWARD FORMANEK
}

(Communicated by Stephen D. Smith)

\begin{abstract}
Let $F$ be a free group of finite rank $n \geq 2$, let $\operatorname{End}(F)$ be the semigroup of endomorphisms of $F$, and let $A u t(F)$ be the group of automorphisms of $F$.

Theorem. If $T: \operatorname{End}(F) \rightarrow \operatorname{End}(F)$ is an automorphism of $\operatorname{End}(F)$, then there is an $\alpha \in \operatorname{Aut}(F)$ such that $T(\beta)=\alpha \circ \beta \circ \alpha^{-1}$ for all $\beta \in \operatorname{End}(F)$.
\end{abstract}

For a group $G$, let $\operatorname{Aut}(G)$ denote the group of automorphisms of $G$, and let $\operatorname{End}(G)$ denote the semigroup of endomorphisms of $G$. Note that $\operatorname{Aut}(G)$ is the group of invertible elements of $\operatorname{End}(G)$, so any automorphism of $\operatorname{End}(G)$ induces an automorphism of $\operatorname{Aut}(G)$ by restriction.

In 1975, J. L. Dyer and the author 2] answered a question of G. Baumslag by proving that if $F$ is a free group of finite rank $n \geq 2$, then $\operatorname{Aut}(F)$ is a complete group; that is, the center of $A u t(F)$ is trivial and every automorphism of $A u t(F)$ is inner. More recently, new proofs and various generalizations of this theorem have been obtained by M. R. Bridson and K. Vogtmann [1, E. Formanek [3], D. G. Khramtsov [4, and V. Tolstykh [5].

While the author was visiting Israel in May, 2000, B. Plotkin asked: What is the structure of the group of automorphisms of the semigroup $\operatorname{End}(F)$ ? Using the completeness of $\operatorname{Aut}(F)$, it is shown below that every automorphism of $\operatorname{End}(F)$ is a conjugation by an element of $A u t(F)$.

Notation. Endomorphisms of $F=F\left\langle x_{1}, \ldots, x_{n}\right\rangle$ will be regarded as functions acting on the left. Since an endomorphism $\alpha: F \rightarrow F$ is completely determined by its values on any free generating set, it can be defined by specifying $\alpha\left(y_{1}\right), \ldots, \alpha\left(y_{n}\right)$, for some free generating set $\left\{y_{1}, \ldots, y_{n}\right\}$ of $F$. The semigroup operation of $\operatorname{End}(F)$ is a composition of functions, denoted "o". Thus $(\alpha \circ \beta)(x)=\alpha(\beta(x))$, and saying that $T$ is an automorphism of $\operatorname{End}(F)$ means that $T: \operatorname{End}(F) \rightarrow \operatorname{End}(F)$ is a bijection satisfying $T(\alpha \circ \beta)=T(\alpha) \circ T(\beta)$, for all $\alpha, \beta \in \operatorname{End}(F)$. Multiplication in $F$ will be denoted by juxtaposition, elements of $F$ will be represented by lower case Roman letters, and elements of $\operatorname{End}(F)$ will be represented by lower case Greek letters.

Received by the editors October 2, 2000.

2000 Mathematics Subject Classification. Primary 20E05.

Key words and phrases. Free group, endomorphism, automorphism.

The author was partially supported by the NSF. 
Theorem. Let $F=F\left\langle x_{1}, \ldots, x_{n}\right\rangle$ be a free group of finite rank $n \geq 2$, and suppose that $T: \operatorname{End}(F) \rightarrow \operatorname{End}(F)$ is an automorphism of the semigroup $\operatorname{End}(F)$. Then there is an $\alpha \in \operatorname{Aut}(F)$ such that $T(\beta)=\alpha \circ \beta \circ \alpha^{-1}$ for all $\beta \in \operatorname{End}(F)$.

Proof. Since $T$ carries $A u t(F)$ to itself, the completeness of $A u t(F)$ [2, Theorem A] implies that there is an $\alpha \in A u t(F)$ such that $T(\beta)=\alpha \circ \beta \circ \alpha^{-1}$ for all $\beta \in A u t(F)$. Replacing $T$ by $T^{\prime}$, where

$$
T^{\prime}(\beta)=\alpha^{-1} \circ T(\beta) \circ \alpha, \text { for all } \beta \in \operatorname{End}(F),
$$

shows that proving the theorem is equivalent to showing

$(*) \quad$ If $T: \operatorname{End}(F) \rightarrow \operatorname{End}(F)$ is an automorphism of $\operatorname{End}(F)$ and

$T(\beta)=\beta$ for all $\beta \in A u t(F)$, then $T(\beta)=\beta$ for all $\beta \in \operatorname{End}(F)$.

Note that if $T$ satisfies the hypotheses of $\left(^{*}\right)$, so does $T^{-1}$, so any property established for $T$ or $T^{-1}$ will also hold for the other.

For $a \in F$, let $\gamma_{a} \in \operatorname{Aut}(F)$ be the inner automorphism of $F$ defined by $\gamma_{a}(x)=$ $a x a^{-1}$, for all $x \in F$. Then for all $\rho \in \operatorname{End}(F), a, x \in F$,

$$
\left(\rho \circ \gamma_{a}\right)(x)=\rho\left(a x a^{-1}\right)=\rho(a) \rho(x) \rho(a)^{-1}=\left(\gamma_{\rho(a)} \circ \rho\right)(x),
$$

so $\rho \circ \gamma_{a}=\gamma_{\rho(a)} \circ \rho$. Now apply $T$, noting that $T\left(\gamma_{a}\right)=\gamma_{a}$, by the hypothesis on $T$ in $(*)$. This gives

$$
T(\rho) \circ \gamma_{a}=T\left(\rho \circ \gamma_{a}\right)=T\left(\gamma_{\rho(a)} \circ \rho\right)=\gamma_{\rho(a)} \circ T(\rho) .
$$

Hence for any $x \in F$,

$$
\begin{aligned}
& T(\rho)(a)][T(\rho)(x)][T(\rho)(a)]^{-1}=T(\rho)\left(a x a^{-1}\right)=\left[T(\rho) \circ \gamma_{a}\right](x) \\
& \quad=\left[\gamma_{\rho(a)} \circ T(\rho)\right](x)=\rho(a)[T(\rho)(x)] \rho(a)^{-1},
\end{aligned}
$$

which implies that $\rho(a)^{-1}[T(\rho)(a)]$ centralizes $T(\rho)(F)$, for all $\rho \in \operatorname{End}(F), \quad a \in F$. Since any property established for $T$ also holds for $T^{-1}$, we may replace $T$ by $T^{-1}$. Then substituting $T(\rho)$ for $\rho$ gives

(1) $[T(\rho)(a)]^{-1} \rho(a)$ centralizes $\rho(F)$, for all $\rho \in \operatorname{End}(F), a \in F$.

Now suppose that $\rho \in \operatorname{End}(F)$ is such that $\rho(F)$ is not abelian. Then the centralizer of $\rho(F)$ in $F$ is trivial, so (1) implies that $[T(\rho)](a)=\rho(a)$ for all $a \in F$; i.e., $T(\rho)=\rho$. Thus we have shown that:

(2) If $\rho(F)$ is not abelian, then $T(\rho)=\rho$.

To establish $\left(^{*}\right)$, it remains to show that $T(\rho)=\rho$ for endomorphisms $\rho$ such that $\rho(F)$ is abelian. Abelian subgroups of $F$ are trivial or infinite cyclic. The trivial endomorphism $(\rho(x)=1$, for all $x \in F)$ is characterized by the multiplicative property $\rho \circ \sigma=\rho$ for all $\sigma \in \operatorname{End}(F)$, so it is fixed by $T$. Thus all that remains to be proved is the following:

(3) If $T: \operatorname{End}(F) \rightarrow \operatorname{End}(F)$ satisfies the hypotheses of (*) and $\rho \in \operatorname{End}(F)$ is an endomorphism such that $\rho(F)$ is infinite cyclic, then $T(\rho)=\rho$.

To prove $(3)$, consider the endomorphism $\delta: F \rightarrow F$ defined by $\delta\left(x_{1}\right)=$ $x_{1}, \delta\left(x_{2}\right)=\delta\left(x_{3}\right)=\ldots=\delta\left(x_{n}\right)=1$. The centralizer of $\delta(F)=g p\left\langle x_{1}\right\rangle$ is $g p\left\langle x_{1}\right\rangle$ itself, so (1) implies that $[T(\delta)(a)]^{-1} \delta(a) \in g p\left\langle x_{1}\right\rangle$ for all $a \in F$. Hence there are integers $i_{1}, \ldots, i_{n}$ such that $T(\delta)\left(x_{j}\right)=x_{1}^{i_{j}}$, for $j=1, \ldots, n$.

For $k=2, \ldots, n$, let $\sigma_{k}$ be the automorphism of $F$ defined by

$$
\sigma_{k}\left(x_{1}\right)=x_{1} x_{k}, \sigma_{k}\left(x_{j}\right)=x_{j} \quad(j=2, \ldots, n) .
$$


Then $\delta \circ \sigma_{k}=\delta$, so

$$
T(\delta) \circ \sigma_{k}=T(\delta) \circ T\left(\sigma_{k}\right)=T\left(\delta \circ \sigma_{k}\right)=T(\delta)
$$

and

$$
x_{1}^{\left(i_{1}+i_{k}\right)}=T(\delta)\left(x_{1} x_{k}\right)=\left[T(\delta) \circ \sigma_{k}\right]\left(x_{1}\right)=T(\delta)\left(x_{1}\right)=x_{1}^{i_{1}},
$$

so $i_{k}=0$ for $k=2, \ldots, n$. Since $\delta \circ \delta=\delta$,

$$
x_{1}^{i_{1}}=T(\delta)\left(x_{1}\right)=[T(\delta) \circ T(\delta)]\left(x_{1}\right)=x_{1}^{i_{1}^{2}} .
$$

Thus $i_{1}^{2}=i_{1}$, so $i_{1}=0$ or $i_{1}=1$. The possibility that $i_{1}=0$ is excluded since $T(\delta)$ would be the trivial endomorphism $(T(\delta)(F)=1)$, which we already know is fixed by $T$. Thus $i_{1}=1$, so $T(\delta)=\delta$.

Finally, suppose that $\rho \in \operatorname{End}(F)$, and that $\rho(F)=g p\langle w\rangle$, an infinite cyclic group. There is a free basis $\left\{y_{1}, \ldots, y_{n}\right\}$ for $F$ such that $\rho\left(y_{1}\right)=w, \rho\left(y_{2}\right)=\rho\left(y_{3}\right)=$ $\ldots=\rho\left(y_{n}\right)=1$. Let $\sigma$ be the automorphism of $F$ defined by $\sigma\left(x_{i}\right)=y_{i}, \quad(i=$ $1, \ldots, n)$, and let $\tau \in \operatorname{End}(F)$ be defined by $\tau\left(y_{1}\right)=w, \tau\left(y_{2}\right)=\ldots=\tau\left(y_{n}\right)=z$, where $z$ is chosen so that $g p\langle w, z\rangle$ is free of rank two. Computing the images of $y_{1}, \ldots, y_{n}$ shows that $\rho=\tau \circ \sigma \circ \delta \circ \sigma^{-1}$. Since $T$ fixes $\tau, \sigma$, and $\delta$, it also fixes $\rho$, which establishes (3) and completes the proof of the theorem.

\section{REFERENCES}

[1] M. R. Bridson and K. Vogtmann, Automorphisms of automorphism groups of free groups, J. Algebra 229 (2000), 785-792. MR 2001g:20041

[2] J. L. Dyer and E. Formanek, The automorphism group of a free group is complete, J. London Math. Soc. 11 (1975), 181-190. MR 52:588

[3] E. Formanek, Characterizing a free group in its automorphism group, J. Algebra 133 (1990), 424-432. MR 92a:20034

[4] D. G. Khramtsov, Completeness of groups of outer automorphisms of free groups (Russian). Group-theoretic Investigations (Russian), 128-143, Akad. Nauk SSSR Ural. Otdel, Sverdlovsk, 1990. MR 94c:20066

[5] V. Tolstykh, The automorphism tower of a free group, J. London Math. Soc. 61 (2000), 423-440. MR 2001c:20081

Department of Mathematics, The Pennsylvania State University, University Park, PenNSYlvania 16802

E-mail address: formanek@math.psu.edu 